\title{
SHORT-TERM EFFECTS OF POSITIVE EXPIRATORY AIRWAY PRESSURE IN PATIENTS BEING WEANED FROM MECHANICAL VENTILATION
}

\author{
Marcelo de Mello Rieder, Alexandre Doval da Costa, Silvia Regina Rios Vieira
}

doi: 10.1590/S1807-59322009000500006

Rieder M de M, da Costa AD, Vieira SRR. Short-term effects of positive expiratory airway pressure in patients being weaned from mechanical ventilation. Clinics. 2009;64(5):403-8.

OBJECTIVE: To investigate the feasibility and the cardiorespiratory effects of using positive expiratory airway pressure, a physiotherapeutic tool, in comparison with a T-tube, to wean patients from mechanical ventilation.

METHODS/DESIGN: A prospective, randomized, cross-over study.

SETTING: Two intensive care units.

PATIENTS AND INTERVENTIONS: We evaluated forty patients who met weaning criteria and had been mechanically-ventilated for more than 48 hours, mean age 59 years, including 23 males. All patients were submitted to the T-tube and Expiratory Positive Airway Pressure devices, at $7 \mathrm{~cm} \mathrm{H} \mathrm{H}_{2} \mathrm{O}$, during a 30-minute period. Cardiorespiratory variables including work of breathing, respiratory rate (rr), peripheral oxygen saturation $\left(\mathrm{SpO}_{2}\right)$, heart rate $(\mathrm{hr})$, systolic, diastolic and mean arterial pressures (SAP, DAP, MAP) were measured in the first and thirtieth minutes. The condition was analyzed as an entire sample set $(n=40)$ and was also divided into subconditions: chronic obstructive pulmonary disease $(\mathrm{n}=14)$ and non-chronic obstructive pulmonary disease (non- chronic obstructive pulmonary disease) $(\mathrm{n}=26)$ categories. Comparisons were made using a t-test and Analysis of Variance. The level of significance was $\mathrm{p}<0.05$.

RESULTS: Our data showed an increase in work of breathing in the first and thirtieth minutes in the EPAP condition $(0.86 \pm 0.43$ and $1.02 \pm 1.3)$ as compared with the T-tube condition $(0.25 \pm 0.26$ and $0.26 \pm 0.35)(\mathrm{p}<0.05)$, verified by the flow-sensor monitor (values in J/L). No statistical differences were observed when comparing the Expiratory Positive Airway Pressure and T-tube conditions with regard to cardiorespiratory measurements. The same result was observed for both chronic obstructive pulmonary disease and non- chronic obstructive pulmonary disease subconditions.

CONCLUSIONS: Our study demonstrated that, in weaning patients from mechanical ventilation, the use of a fixed level of Expiratory Positive Airway Pressure caused an increase in work of breathing that was not accompanied by any other significant cardiorespiratory changes. Therefore, we have to be cautious when using Expiratory Positive Airway Pressure as a physiotherapeutic tool during weaning from mechanical ventilation.

KEYWORDS: Positive Expiratory Airway Pressure; Weaning; Mechanical Ventilation; Intensive Care Unit; Physiotherapy.

\section{INTRODUCTION}

The discontinuation or withdrawal process from mechanical ventilation is an important clinical challenge. Up to $40 \%$ of the time spent during mechanical ventilation

Intensive Care Unit, Hospital de Clinicas de Porto Alegre - Porto Alegre/ RS, Brazil.

Email: mdrieder@brturbo.com.br

Tel.: 55512101.8000

Received for publication on December 14, 2008

Accepted for publication on February 09, 2009 is devoted to weaning, which is described as the process of gradual and progressive removal of ventilatory support. ${ }^{1,2}$ Formal discontinuation assessment for patients who receive mechanical ventilation for respiratory failure should be performed during spontaneous breathing rather than while the patient is still receiving substantial ventilatory support. An initial brief period of spontaneous breathing can be used to assess the advisability of continuing onto a formal Spontaneous Breathing Trial (SBT). ${ }^{3-5}$

The most important mechanisms that lead to unsuccessful weaning include alterations in respiratory drive, impaired 
oxygenation, and excessive load being imposed on respiratory muscles. ${ }^{6}$ Certain studies suggest that the application of external Positive Expiratory Pressure (PEP) during mechanical ventilation minimizes hyperinflation effects and reduces the work of breathing, which may be helpful during the weaning process. ${ }^{7}$

Positive Expiratory Airway Pressure (EPAP) is a technique that has been used as an effective and safe alternative to chest physiotherapy. During EPAP therapy, the patient exhales against a threshold resistor, generating preset positive expiratory pressures. The purpose of this therapy is to increase the transpulmonary pressure gradient and improve pulmonary expansion, which consequently improves oxygenation. ${ }^{89}$ EPAP can mobilize secretions, help in the management of post-operative atelectasis, and enhance the benefits of aerosol bronchodilator delivery. It is also known that EPAP and Continuous Positive Airway Pressure (CPAP) can reduce inspiratory load, possibly secondary to a reduction in hyperinflation. ${ }^{10}$ Hyperinflation with a decrease in inspiratory capacity is a common presentation in some patients, such as those with asthma and COPD and there is some evidence advocating the use of EPAP in Intensive Care Units (ICU) to reduce air trapping in those patients. ${ }^{11}$ To our knowledge, no research to date has compared the use of EPAP and T-tube devices in weaning patients.

This study aims to investigate the feasibility of EPAP as a physiotherapy tool in weaning patients from mechanical ventilation, evaluating its short term hemodynamic and respiratory effects.

\section{MATERIALS AND METHODS}

\section{Study Population}

This study took place between January 2005 and July 2007, in two Intensive Care Units (ICU). The sample condition was composed of 40 critically ill patients who had been on mechanical ventilation for more than 48 hours, with different causes of respiratory failure. Their clinical characteristics are displayed in Table 1.

Patients considered fit for weaning were selected by ICU physicians on the basis of the routinely applied ICU criteria, which included improvement or resolution of the cause of respiratory failure; adequate gas exchange, indicated by $\mathrm{PaO}_{2}$ $/ \mathrm{FiO}_{2} \geq 200$ or $\mathrm{PaO}_{2} \geq 60 \mathrm{mmHg}$; oxygen saturation $\geq 90 \%$, $\mathrm{FiO}_{2} \leq 0.40$, with PEEP $\leq 5 \mathrm{cmH}_{2} \mathrm{O}$; Glasgow Coma Score $\geq$ 13; absence of hyperthermia; acceptable hemoglobin levels; respiratory rate $(\mathrm{RR})<35$ breaths/min; tidal volume $\left(\mathrm{V}_{\mathrm{T}}\right)$ on non-assisted breathing $>5 \mathrm{~mL} / \mathrm{kg}$; maximum inspiratory pressure $(\mathrm{PI} \max ) \leq-20 \mathrm{cmH}_{2} \mathrm{O}$; and hemodynamic stability without the use of vasoactive drugs (dopamine, dobutamine
Table 1 - Patient Clinical Features

\begin{tabular}{lc}
\hline Characteristics & \\
Mean Age (years) \pm SD & $59.4 \pm 18.1$ \\
Gender N $(\%)$ & $23(57.5)$ \\
Male & $17(42.5)$ \\
Female & $13.3 \pm 10.4$ \\
Mean ICU admission APACHE II \pm SD & \\
Mechanical Ventilation durations (days) & N (\%) \\
$<5$ & $13(32.5)$ \\
5 - 10 & $13(32.5)$ \\
$>10$ & $14(35.0)$ \\
COPD & \\
COPD & $14(35.0)$ \\
Non-COPD & $26(65.0)$ \\
Etiology of Respiratory Failure N (\%) & \\
Acute Respiratory Distress Syndrome (ARDS) & $10(25.0)$ \\
Acute Pulmonary Edema (APE) & $8(20.0)$ \\
COPD & $8(20.0)$ \\
Coma & $4(10.0)$ \\
Pneumonia & $3(7.5)$ \\
Post-op & $3(7.5)$ \\
Cardiopulmonary Resuscitation (CPR) & $3(7.5)$ \\
Guillian-Barré Syndrome & $1(2.5)$ \\
\hline
\end{tabular}

$\mathrm{SD}=$ Standard Deviation; ICU = Intensive Care Unit; APACHE = Acute Physiology and Chronic Health Evaluation II; COPD = chronic obstructive pulmonary disease; ARDS = Acute Respiratory Distress Syndrome; APE = acute pulmonary edema; Post-op $=$ Postoperative $; \mathrm{CPR}=$ Cardiopulmonary Resuscitation.

or norepinephrine) or sedatives. Exclusion criteria included patients with hypotension (systolic blood pressure $<100$ $\mathrm{mmHg}$ ) due to hypovolemia or direct cardiovascular involvement; patients using vasoactive drugs (dopamine, dobutamine or norepinephrine); patients with severe intracranial disease, with barotrauma and/or chest tubes; and those using sedatives. This study was approved by the ethics committees of both hospitals. All participating patients or their relatives signed an informed consent form.

\section{Design}

This study was a cross-over randomized clinical trial. Each patient was observed during the use of both T-tube (control) and EPAP methods. The sequence in which the methods were implemented was determined by random selecting sealed envelopes.

All patients entering the study were ventilated either on Servo 900C or on Servo 300C (Siemens-Elema, Solna, Sweden) systems under low levels of Pressure Support 
(PS) $<12 \mathrm{cmH}_{2} \mathrm{O}$, before commencing the protocol. The following information on each patient's clinical status was registered in standard formats: age, sex, APACHE Scale II (Acute Physiology and Chronic Health Evaluation II), and time of mechanical ventilation.

Mechanical ventilation parameters registered prior to protocol commencement included ventilation mode, PS level, Fractional Concentration of Oxygen $\left(\mathrm{FiO}_{2}\right)$ Tidal Volume $\left(\mathrm{V}_{\mathrm{T}}\right)$, Respiratory Rate $(\mathrm{RR})$, and peak inspiratory pressure (PIP). Arterial blood gas analysis, performed prior to our protocol, was also recorded. Patients were then submitted to either T-tube or EPAP treatment in a random order.

During T-tube treatment, the patient was disconnected from mechanical ventilation, and an oxygen flow of $5 \mathrm{~L} / \mathrm{min}$ was added to the endotracheal tube (ETT) or tracheostomy. Inspiratory and expiratory breathing efforts were made spontaneously by the patient. Oxygen flow added to the T-tube was kept at $5 \mathrm{~L} / \mathrm{min}$.

During EPAP, patients breathed spontaneously through the EPAP system adapted for either the ETT or tracheostomy. The EPAP system consisted of a T-tube with a unidirectional inspiratory valve and a spring-loaded expiratory valve. Pressure was maintained at $7 \mathrm{cmH}_{2} \mathrm{O}$, the same value used during PS by other authors. Oxygen flow was $5 \mathrm{~L} / \mathrm{min}^{12,13}$

\section{Data Collection}

To monitor ventilatory mechanics, a Ventrak Model 1500 (Novametrix Medical Systems, Wallingford, CT, USA) was used. This device continuously processes readings from a flow sensor (Disposable Flow Sensor, cat. № 6038, Novametrix Medical Systems, Wallingford, CT, USA) that is positioned between the end of the ETT or tracheostomy and the T-tube, both with or without EPAP. The flow sensor converts gas flow into a linear signal, identifying the differences in circuit flow and pressure. The Ventrak 1500 is attached to a portable IBM-compatible computer (Fujitsu, mod. 756 DX, Japan) via a serial port. We used software supplied by Novametrix for data storage and subsequent analysis.

Pressure on the ETT and tracheostomy was checked at the onset of Ventrak 1500 measurements in order to ensure there was no leakage of exhaled air, which may impair the recording of variables. The Ventrak 1500 is supplied with its own flow and pressure calibration software, which we used at the beginning of each monitoring period. Calibration was performed with a $100 \mathrm{~mL}$ Calibration Syringe. (Model No 5510, Hans Rudolph, Kansas City, Mo., USA). Variables recorded by the Ventrak system included work of breathing $\left(\mathrm{WOB}_{\text {total }}=\mathrm{J} / \mathrm{L}\right)$ and tidal volume.

The HP Multiparametric Modular Model 66S Monitor (Hewlett-Packard USA) was used for non-invasive measurements of hemodynamic parameters as well as to monitor parameters related to gas exchange. This monitor is routinely used for inpatients in ICUs. The recorded parameters were heart rate $(\mathrm{HR}=\mathrm{bpm})$; systolic, diastolic, and mean arterial pressures (SAP, DAP, $\mathrm{MAP}=\mathrm{mmHg}$ ); and peripheral oxygen saturation $\left(\mathrm{SpO}_{2}=\%\right)$. All of these parameters were recorded by a portable computer at the times designated by our protocol.

The protocol was initiated and the patient was transferred from previous ventilation parameters in PS to the first method, as determined by randomization. Parameters were registered at Time 1: one minute after the installation of the designated weaning mode, and at Time 30: 30 minutes after the first measurement. Thirty minutes after the beginning of the first weaning method, the patient was put back on mechanical ventilation for at least 30 minutes, under the same ventilation parameters used prior to the beginning of the protocol. This period was intended to allow for respiratory rest and to restore the parameters to which the patient had been exposed before the beginning of the protocol. Thereafter, the second weaning method was tested.

As described in the literature, the criteria for interrupting our protocol included signs of respiratory distress, a drop in oxygen saturation $(<88 \%)$, an increase in the respiratory rate ( $>35 \mathrm{bpm}$ ) and heart rate (> $140 \mathrm{bpm}), \mathrm{SAP}>180 \mathrm{mmHg}$ or $<90 \mathrm{mmHg}$, agitation, and a change or reduction in the degree of alertness.

\section{Data Analysis}

Initially, a general analysis of the entire sample condition $(n=40)$ was performed, as well as an analysis of each condition under the two treatment methods at the various time points. Variables were analyzed by comparing T-tube (control) and EPAP treatments at the first and thirtieth minutes.

Patients were also analyzed in subconditions to compare COPD versus non-COPD patients. Stata 8.0 was used for data storage and analysis. Results are represented in terms of means and standard deviations for continuous variables that are normally distributed, and as ratios for categorical variables. Comparisons were made using the Student t-test for paired samples. Significance was established at $\mathrm{p}<0.05$.

\section{RESULTS}

The clinical characteristics of the 40 enrolled patients are shown in Table 1. All patients completed the protocol.

Mechanical ventilation parameters of patients before they entered the protocol were PS level $=10 \pm 2 \mathrm{cmH}_{2} \mathrm{O}$, PEEP 
$=5 \pm 1 \mathrm{cmH}_{2} \mathrm{O}, \mathrm{FiO}_{2}=38 \pm 2, \mathrm{~V}_{\mathrm{T}}=525 \pm 155 \mathrm{ml}$, and $\mathrm{RR}=$ $20 \pm 5 \mathrm{rpm}$.

Arterial blood gas analysis performed within one hour prior to commencing the protocol showed $\mathrm{pH}=7.43 \pm 0.2$, $\mathrm{PaCO}_{2}=35 \pm 8, \mathrm{HCO}_{3}=24 \pm 2, \mathrm{PaO}_{2}=96 \pm 15$, and oxygen saturation $=97 \pm 3 \%$.

\section{Hemodynamic and Respiratory Measurements}

With regard to respiratory rate, we observed an increase between the first and the thirtieth minutes with the EPAP method $(\mathrm{p}<0.01)$ (Table 2). No differences were observed when comparing COPD and non-COPD, and tracheo and non-tracheo categories.

No significant differences were identified in terms of heart rate, mean arterial pressure and peripheral oxygen saturation.

Analysis of respiratory effort and comparisons between the EPAP and T-tube methods showed a greater WOB total within the EPAP condition, when comparing across the entire condition as well as between all COPD and nonCOPD subconditions, in both the first and thirtieth minutes (Figures 1, 2 and 3).

\section{Work of Breathing in the study population}

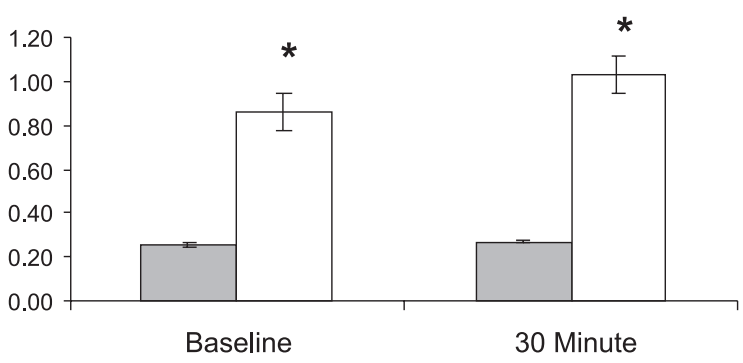

$\square$ T tube

$\square$ EPAP

Figure 1 - Work of Breathing in the study population: Comparison between $\mathrm{EPAP}$ and T-tube methods. EPAP $=$ Positive Expiratory Airway Pressure. Values expressed in Joules/L. * Student $t$-test comparing EPAP ant T-tube methods: $p<0.05$

\section{DISCUSSION}

Our results suggest that EPAP caused an increase in the work of breathing. No differences were observed for the other hemodynamic and respiratory parameters. These observations were made by analyzing the entire

Table 2 - Hemodynamic and Respiratory Measurements: Comparison between EPAP and T-tube methods.

\begin{tabular}{|c|c|c|c|c|}
\hline & EPAP & p-value $*$ & T-tube & p-value * \\
\hline & 0 - 30 minutes & & 0 - 30 minutes & \\
\hline HR ALL & $96.2(17.9)-99(18.2)$ & NS & 89.9 (21.3)-98.0 (22.8) & NS \\
\hline HR COPD, bpm & $88.2(18.1)-91.5(29.5)$ & NS & 93.7 (14.7)-95.6 (18.0) & NS \\
\hline HR non-COPD, bpm & $97.46(19.5)-100.9(18.1)$ & NS & $93.5(18.8)-99.0(17.0)$ & NS \\
\hline $\mathrm{p}$-value $* * *$ & & NS & & NS \\
\hline MAP ALL & $96.4(12.5)-97.1$ (13.2) & NS & 94.4 (11.8)-96.3 (12.2) & NS \\
\hline MAP COPD, mmHg & 93.5 (14.7)-92.6 (1.4) & NS & 95. (12.7)-94.1 (11.1) & NS \\
\hline MAP non-COPD, mmHg & $94.0(20)-95.5(21.1)$ & NS & 94.8 (10.1)-98.3 (11.8) & NS \\
\hline $\mathrm{p}$-value $* * *$ & & NS & & NS \\
\hline RR ALL & $24.7(6.1)-28.8(8.1)$ & $\mathrm{p}<0.01$ & $26.8(7.9)-27.6(6.9)$ & NS \\
\hline RR COPD, rpm & $24.3(5.1)-28.7(6.6)$ & NS & $28.3(8.7)-27.6(6.5)$ & NS \\
\hline RR non-COPD, rpm & $23.8(6.5)-28.8(8.9)$ & $\mathrm{p}<0.01$ & $25.9(7.4)-27.6(7.2)$ & NS \\
\hline $\mathrm{p}$-value $* * *$ & & NS & & NS \\
\hline SpO2 ALL & $96.1(3.1)-95.6(3.9)$ & NS & $95.3(2.5)-94.8(3.7)$ & NS \\
\hline $\mathrm{SpO} 2 \mathrm{COPD}, \%$ & $96.5(2.9)-96(4.1)$ & NS & $95.7(2.3)-94.4(3.7)$ & NS \\
\hline $\mathrm{SpO} 2$ non-COPD, \% & $95.8(3.2)-95.3(3.8)$ & NS & $95.3(2.6)-95.0(3.7)$ & NS \\
\hline $\mathrm{p}$-value $* * *$ & & NS & & NS \\
\hline
\end{tabular}

Comparison between EPAP and T-tube methods. EPAP= Positive Expiratory Airway Pressure; HR = Heart Rate; MAP = mean arterial pressure; RR = Respiratory Rate; $\mathrm{SpO}_{2}=$ peripheral oxygen saturation; COPD = Chronic Obstructive Pulmonary Disease; non-COPD = non Chronic Obstructive Pulmonary Disease. * Student t-test comparing times 1 and 30 ;

** Student t-test comparing times 1 and 30;

*** Student t-test comparing COPD and non-COPD. Values expressed as means and standard deviations

Analysis of respiratory work comparing EPAP and T-tube methods showed a greater WOB total under the EPAP method, within the entire condition as well as between all COPD and non-COPD subconditions, both in the first and the thirtieth minutes (Figures 1, 2 and 3) 

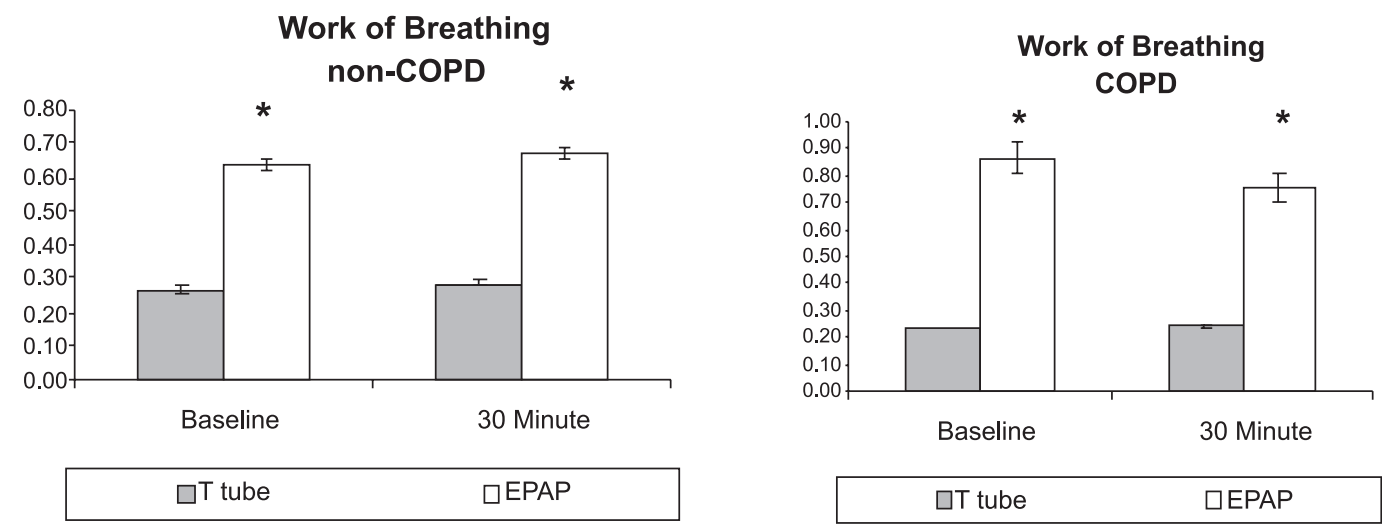

Figure 2 and 3 - Work of Breathing: Comparison between EPAP and T-tube methods in subconditions COPD and non-COPD. EPAP= Positive Expiratory Airway Pressure; $\mathrm{COPD}=$ Chronic Obstructive Pulmonary Disease; non-COPD = non Chronic Obstructive Pulmonary Disease. Values expressed in Joules/L. * Student t-test comparing EPAP ant T-tube: $p<0.05$

sample condition as well as the COPD and non-COPD subconditions.

It is important to note that EPAP is used by physiotherapists in certain situations but has not been evaluated to date in the context of weaning patients from mechanical ventilation. Some of its beneficial effects in preventing atelectasis and in clearing secretions may be useful in this context, because it is known that supplementary EPAP is useful in mobilizing secretions and treating atelectasis. ${ }^{5,9,13} \mathrm{We}$ also know that other forms of PEP are helpful in preventing lung collapse and in removing pulmonary secretions. Recently, Positive Airway Pressure (PAP) devices have been used to treat patients with sleeprelated breathing disorders. ${ }^{10} \mathrm{CPAP}$ has been shown to be associated with reinsufflation of collapsed lung areas and may exert a potential bronchial cleaning effect, associated with an increase in transpulmonary pressure. ${ }^{14,15}$ PEP was associated with increases in lung volume and removal of pulmonary secretions. The most likely explanation for these beneficial effects of expiratory pressure is the improvement of lung collateral ventilation and probably a reduction in air trapping. These effects in the context of positive airway pressure may be beneficial in reducing respiratory work.

However, the implementation of CPAP requires a flow system that incorporates specific flow-generating valves or supplementary hardware in the mechanical respirator itself. Our EPAP study aimed to evaluate a low-cost, spontaneous breathing system. We wished to achieve beneficial effects similar to those associated with CPAP, but we failed to meet these goals.

Comparison between T-tube and EPAP methods showed no significant differences in hemodynamic measurements and $\mathrm{SpO}_{2}$. Therefore, we suggest that these approaches are not harmful, at least in terms of oxygenation. However, there was a significant increase in WOB during EPAP use.

Significant increases in WOB totals were related to increased respiratory work under the EPAP protocol, as compared to the T-tube method. There are no data in the literature regarding such a comparison. Measurement of WOB is quite complex and is better reproduced by experimental models. Nevertheless, measurements can be recorded in patients with spontaneous, assisted, or controlled breathing. ${ }^{16}$ WOB totals reflect the effort needed to overcome elastic forces (compliance) of the pulmonary parenchyma and of the thoracic wall, as well as viscous forces (resistance) attributed to the airways, lungs and thoracic wall tissues. ${ }^{16}$ WOB measurements reflect the strength of an individual's respiratory muscles in regard to the reserves needed to return to spontaneous respiration (patient work). ${ }^{16-19}$ WOB is commonly increased in critically ill patients, but many patients have a decreased capacity for working performance. The level of expiratory pressure used during the EPAP method, even though it was low, imposed an increase in the expiratory resistance. Consequently, there was also a substantial inspiratory load, probably due to an increase in negative intrapleural pressure generated before the commencement of inspiratory flow. This may explain the increase in WOB as well as in respiratory rate in the EPAP condition. Nevertheless, a very important point in this study is the fact that a greater WOB was observed in all the subconditions studied.

Regarding limitations of this study, we wish to highlight the small number of subjects and we note the heterogeneity of diseases causing respiratory failure that can cause different responses. It is also important to point out that no invasive monitoring strategy was used because all patients had their respiratory failure adequately controlled and were not only being weaned from mechanical ventilation, but also from more aggressive monitoring systems.

Nevertheless, the greater WOB data was observed in all subconditions studied, and we think this result is of interest. 
In conclusion, our study demonstrated that in weaning patients from mechanical ventilation, the use of a fixed level of positive expiratory airway pressure caused an increase in WOB without other significant cardiorespiratory changes.
Therefore, we must be cautious when using EPAP as a physiotherapeutic tool during weaning from mechanical ventilation.

\section{REFERENCE}

1. Esteban A, Alía I, Ibañez J, Benito S, Tobin MJ. Modes of mechanical ventilation and weaning. A National Survey of Spanish hospitals. Spanish Lung Failure Collaborative Condition. Chest. 1994;106;118893.

2. Alvisi R, Volta CA, Righini ER, Capuzzo M, Ragazzi R, Verri M, et al Predictor of weaning outcome in chronic obstrutive pulmonary disease. Eur Respir J. 2000;656-62.

3. Frutos-Vivar F, Esteban A. When to wean from a ventilator: an evidencebased strategy. Cleve Clin J Med. 2003; 70;389,392-3,397.

4. Lessard MR, Brochard LJ. Weaning from mechanical ventilatory support. Clin Chest Med. 1996 ;17:475-89.

5. MacIntyre NR, Cook DJ, Ely EW Jr, Epstein SK, Fink JB, Heffner $\mathrm{JE}$, et al. Evidence-based guidelines for weaning and discontinuing ventilatory support: a collective task force facilitated by the American College of Chest Physicians; the American Association for Respiratory Care; and the American College of Critical Care Medicine. Chest. 2001 Dec;120(6 Suppl):375S-95S.

6. MacIntyre NR. Respiratory Mechanics in the Patient Who Is Weaning From the Ventilator. Respir Care. 2005 Feb;50(2):275-86; discussion 284-6.

7. Tobin, MJ, Lodato RF. PEEP, auto-PEEP, and waterfalls (editorial) Chest, 1989;96;449-51.

8. AARC clinical practice guideline. Use of positive airway pressure adjuncts to bronchial hygiene therapy. American Association for Respiratory Care. Respir Care. 1993;38;516-21.

9. Fink JB. Positive Pressure Techniques for Airway Clearance. Respir Care. 2002;47;786-96.

10. Kushida CA, Chediak A, Berry RB, Brown LK, Gozal D, Iber C, et al. Clinical guidelines for the manual titration of positive airway pressure in patients with obstructive sleep apnea. J Clin Sleep Med. 2008;4;15771 .
11. Gattinoni L, Pelosi P, Crotti S, Valenza F. Effects of positive endexpiratory pressure on regional distribuition of tidal volume and recruitment in adult respiratory distress syndrome. Am J Respir Crit Care Med. 1995;151;1807-14.

12. Ferrer M, Esquinas A, Arancibia F, Bauer TT, Gonzalez G, Carrillo A, et al. Noninvasive ventilation during persistent weaning failure: a randomized controlled trial. Am J Respir Crit Care Med. 2003;168;706.

13. Smith TC, Marini JJ. Impact of PEEP on lung mechanics and work of breating in severe airflow obstruction: the effect of PEEP on auto-PEEP. J Appl Physiol. 1988; 65;1488-99.

14. Andersen JB, Qvist H, Kann T. Recruiting collapsed lung trough collateral channels with positive end-expiratory pressure. Scan J Respir Dis. 1979;60;260-6.

15. Main E, Prasad A, Van der Schans C. Conventional chest physiotherapy compared to other airway clearance techniques for cystic fibrosis. Cochrane Database Syst Rev. 2005 25;CD002011.

16. Blanch PB, Banner M. A new respiratory monitor thet enables accurate measurement of work of breathing: A validation study. Respir Care. 1994;897-905

17. Bikker IG, van Bommel J, Reis Miranda D, Bakker J, Gommers D. Endexpiratory lung volume during mechanical ventilation: a comparison with reference values and the effect of positive end-expiratory pressure in intensive care unit patients with different lung conditions. Crit Care. $2008 ; 20 ; 12 ; 145$

18. Brochard L. Intrinsic (or auto-) PEEP during controlled mechanical ventilation. Intensive Care Med. 2002;28; 1376-8.

19. Caruso P, Carnieli DS, Kagohara KH, Anciães A, Segarra JS, Deheinzelin D. Trend of maximal inspiratory pressure in mechanically ventilated patients: predictors. Clinics. 2008;63:33-8. 